triggered $\mathrm{EBV}$ reactivation provokes nuclear architecture remodeling in B-cells and induces spatial proximity (colocalization) of MYC and IGH loci. In theory, the closer the two loci are, the higher the probability of translocation between them is. Methods: 3D FISH was used to study MYC and IGH loci proximity in B-cell lines, in naïve B-cells from healthy donors and in B-cells from EBV-infected African children. These children were divided into three groups according to their infection status (no infection, latency and reactivation). We used an EBV-positive B-cell line inducibly expressing Cas9 and gRNAs targeting MYC and IGH loci to study the consequence of prolonged MYC-IGH proximity on the occurrence of BL characteristic translocation $\mathrm{t}(8 ; 14)$. After induction of DSBs in the targeted loci, the translocation $(8 ; 14)$ occurs with a frequency of $2 \times 10-3$ and is detectable by qPCR. Results: We demonstrated that EBV reactivation by various methods in our model significantly increases MYC-IGH colocalization rate. The same effect was not observed in B-cells from healthy donors treated with Zebra suggesting involvement of EBV in this process. Also, in children, no difference was observed between the latency and reactivation groups, probably due to the very low percentage of EBV-infected B-cells in vivo (less than $1 \%$ ). Then, exploiting our model, we found that EBV reactivation increased both MYC-IGH proximity and translocation rate when DSBs were induced on MYC and IGH. Conclusion: Our work provides the first experimental proof that spatial proximity loci increases the probability of translocations between these loci and explains the link between EBV and specific chromosomal translocations leading to BL. doi: http://dx.doi.org/10.7124/bc.0009F7

\section{S-3. The topological relationship between ribogenesis, mRNA transcription/splicing and the tension of actin cytoskeleton}

Kristine Salmina $^{1}$, Reet Kurg${ }^{2}$, Jekabs

Krigerts $^{1}$, Jekaterina Erenpreisa ${ }^{1}$

${ }^{1}$ Latvian Biomedical Research and Study Centre, Latvia; ${ }^{2}$ Institute of Technology, University of Tartu, Estonia salmina.kristine@gmail.com

To study the topological relationship between the nucleolar fibrillar centres, perinucleolar heterochromatin, nuclear speckles (splicesomes) and the lamin- associated heterochromatin after RNA transcription inhibition. Methods: MCF7 breast cancer cells were treated in 3D preserving conditions by increasing concentration/time of Actinomycin D (AcD). Immunofluorescent staining, confocal microscopy, and image analysis were performed. Results: In control cells with active rRNA and mRNA synthesis, the perinucleolar repressive heterochromatin labelled by $\mathrm{H} 3 \mathrm{~K} 9 \mathrm{Me} 3 /$ cen forms extended structures bent around nucleoli, speckles located more externally are also extended, lamin B1 ideally outlines the nuclear envelope (NE), while actin filaments form fibrils both circular around NE and perpendicular or at angles to it, attached to the cellular membrane. When the nucleolar synthesis is initially suppressed by low AcD, the remnant Pol I cofactor RPA194 forms a few large granules at the nucleolar margin, $\mathrm{H} 3 \mathrm{~K} 9 \mathrm{Me} 3$ heterochromatin condenses in round clumps between them, while speckles also condense as regular circular structures 
around the latter - all together revealing a radial-concentric order. The lamin B1-positive nuclear contour becomes irregular and lamin forms folds in nuclear interior reaching the nucleoli. Actin ring around the nucleus becomes thicker, while its extending cytoplasmic fibers less tense. Full suppression of both syntheses by high dosage/prolong AcD or a-amanitin brings to disorganization of the radialconcentric nuclear order. Conclusions: The links of the perinucleolar and lamin-associated heterochromatin with inner nuclear compartments are involved in topological coordination between the ribogenesis and mRNA maturation, where the radial tension of the actin cytoskeleton exerted via concentric elasticity of the nuclear lamin is part of this regulation.

doi: http://dx.doi.org/10.7124/bc.0009F8

\section{S-4. Exploring the features of Burkitt's lymphoma-associated $t(8 ; 14)$ translocations generated via a CRISPR/Cas9-based system}

\author{
A. A. Shmakova ${ }^{1,2}$, D. Germini ${ }^{1,2}$, Y. S. Vas- \\ setzky ${ }^{1,2,3}$ \\ ${ }^{1}$ UMR 8126, CNRS, Univ. Paris-Sud, Institut Gus- \\ tave Roussy, Villejuif, France; ${ }^{2}$ LIA 1066 LFR2O \\ French-Russian Joint Cancer Research Laboratory, \\ Villejuif, France; ${ }^{3}$ Koltzov Institute of Develop- \\ mental Biology, Moscow, Russia \\ anya.shmakova@list.ru
}

Burkitt lymphoma (BL), an aggressive B-cell lymphoma, is most frequently associated with a chromosomal translocation $\mathrm{t}(8 ; 14)$ that relocates the MYC oncogene near the IGH gene locus. Exploring translocation formation mechanisms and approaches to diminish the risk of their formation is a promising aim. We aimed to investigate the kinetics of $t(8 ; 14)$ accumulation in a cell culture and to assess how targeting different cellular pathways affects translocation frequency. Methods: CRISPR/Cas9 was used to generate double-strand breaks (DSBs) in the IGH and MYC gene loci in a human B-cell line. We evaluated the $\mathrm{t}(8 ; 14)$ level 48 hours, two, three, four, five and six weeks after DSBs induction by qPCR [1]. We also analyzed the level of $t(8 ; 14) 48$ hours after the simultaneous DSBs induction and addition of chemical inhibitors to cell medium. We used NU7026 (DNA-PKcs inhibitor, canonical NHEJ), L67 (Lig1/Lig3 inhibitor, alternative NHEJ), sodium azide (electron-transport chain inhibitor). Results: We found that acquiring $\mathrm{t}(8 ; 14)$ does not provide any selective advantage in the overall cell population as the level of $t(8 ; 14)$ progressively declined after DSBs induction. This can be due to increased expression of MYC in cells with $\mathrm{t}(8 ; 14)$, which is known to induce apoptosis via the p53 pathway [2]. When testing the effect of different drugs on translocation efficiency in our in vitro system, we found that sodium azide decreased the level of $t(8 ; 14)$, which can be explained by energy depletion, as DNA repair is an ATP-dependent pathway [3]. We also observed that L67 significantly decreased the level of $t(8 ; 14)$, while NU7026 significantly increased the level of $\mathrm{t}(8 ; 14)$. Conclusions: We revealed that in vitro IGH-MYC translocation provides no selective advantage as the translocation is lost during long cell culturing. We also found that energy depletion and alternative NHEJ inhibition can decrease the level of $\mathrm{t}(8 ; 14)$ translocations. We 